\title{
Unraveling Impacted Maxillary Incisors: The Why, When, and
} How

\author{
Shubhangi Jain ${ }^{1}$, Monis Raza², Payal Sharma ${ }^{3}$, Piush Kumar ${ }^{4}$
}

\begin{abstract}
Failure of eruption of maxillary incisors requires careful diagnosis and treatment planning. The cause of impaction may vary from physical obstruction in the path of eruption, tooth material arch length discrepancy to malformation of the tooth. General principles of management of the condition include removal of physical obstruction, creation of space, and surgical exposure with or without traction. The treatment of an unerupted tooth depends upon its age, position, etiology, and amount of space in the dental arch. This case series elaborates on three different cases of incisor impaction with different etiologies and varying ranges of complexity. Three-dimensional radiography was utilized in all cases to accurately visualize the impacted tooth and its relation to adjacent structures. All the cases required different approaches and were completed in varying time durations. Meticulous treatment planning resulted in well-aligned satisfactory functional and esthetic results.
\end{abstract}

Keywords: Impaction, Incisor, Smile.

International Journal of Clinical Pediatric Dentistry (2021): 10.5005/jp-journals-10005-1903

\section{INTRODUCTION}

The maxillary incisors are crucial to facial esthetics as they are on maximum display during speech and smiling in most individuals. ${ }^{1}$ A missing incisor may also produce a functional problem due to speech difficulties such as the production of "s" sound. ${ }^{2}$ Therefore, the normal eruption, position, and morphology of these teeth are important for normal phonetics and esthetics. The failure of eruption of maxillary incisor usually presents itself in the mixed dentition stage between the ages of 7 years and 9 years. ${ }^{3}$ It is the third most commonly impacted tooth with variable incidence ranging from 0.06 to $0.2 \%{ }^{4}$ Eruption of the maxillary incisor is considered to be delayed if the eruption of the contralateral incisor occurred 6 months earlier, the lower incisors have erupted $>1$ year earlier or there is a deviation from the normal sequence of eruption. ${ }^{2}$

Failure of eruption of maxillary incisors can occur due to pathological obstruction, tooth malformation, the ectopic position of the tooth bud, non-vital or ankylosed primary teeth, endocrine abnormalities, or bone disease. Pathological obstructions can occur in the form of supernumerary teeth, odontomas, cysts, or thick tissue barriers that develop due to early extraction of the primary tooth. ${ }^{5}$ Trauma to the anterior region can result in loss of the deciduous tooth, dilaceration of the permanent incisor, arrested root development, or intrusive luxation of the permanent incisor. Any change in position or change in morphology of the tooth can fail eruption of the tooth. ${ }^{6}$ The degree of damage of the permanent tooth depends on the developmental stage of the tooth in question, as well as the type and direction of the trauma inflicted. Other possible causes of lack of eruption of maxillary incisors are an ectopic position of the tooth bud, non-vital or ankylosed primary teeth, early extraction (or loss) of deciduous teeth endocrine abnormalities, and bone disease. ${ }^{7}$

Diagnosis of impacted incisors comprises of clinical examination to identify retained deciduous teeth and palpation of the alveolar region. The clinician may encounter a painless, incompressible, palatal, or vestibular fibromucosal protuberance. ${ }^{1}$

Intraoral radiographs can confirm a diagnosis. Periapical views and/or an upper standard occlusal radiograph determine the
${ }^{1-4}$ Department of Orthodontics and Dentofacial Orthopaedics, ITS Center for Dental Studies and Research, Ghaziabad, Uttar Pradesh, India

Corresponding Author: Shubhangi Jain, Department of Orthodontics and Dentofacial Orthopaedics, ITS Center for Dental Studies and Research, Ghaziabad, Uttar Pradesh, India, Phone: +91 9868991306, e-mail: shubhangi.jain.92@gmail.com

How to cite this article: Jain $S$, Raza M, Sharma $P$, et al. Unraveling Impacted Maxillary Incisors: The Why, When, and How. Int J Clin Pediatr Dent 2021;14(1):149-157.

Source of support: Nil

Conflict of interest: None

presence and position of maxillary incisor teeth and any underlying developmental anomalies or pathology. The buccolingual position of the unerupted tooth can be localized by the use of horizontal or vertical parallax. ${ }^{8}$ A cephalometric radiograph can be used to assess the height of impaction and buccolingual inclination of the crown and root of the tooth. Cone-beam computed tomography (CBCT) provides a clear three-dimensional view of the impacted teeth and the associated structures. Cone-beam computed tomography is valuable in treatment planning, as the degree of aberrant crownroot angulation can be assessed and imaging used to plan the optimal direction of traction required, ensuring that both the crown and root are maintained in alveolar bone during alignment of the tooth ${ }^{3}$

This article will discuss a few cases of impacted incisors treated with different approaches.

\section{Case Descriptions}

\section{Case 1}

A 9-year-old female in mixed dentition was reported with a chief complaint of missing upper front tooth. The patient had a convex profile and asymmetric smile. Intraorally, the patient had a class 1 molar relationship bilaterally with crowding in maxillary and 
mandibular arch (Fig. 1). Three supernumerary teeth between upper right central incisor and upper left lateral incisor teeth had been diagnosed radiographically and surgically removed 5 months earlier. The maxillary left central incisor was impacted and the adjacent teeth had drifted into the unoccupied space. The impacted tooth was tipped mesially with the crown height at the middle third of the adjacent incisor (Fig. 2). The cause of impaction was established as an obstruction to the path of eruption due to supernumerary teeth.

The follow-up radiograph taken 5 months after extraction of supernumerary tooth showed no improvement of the vertical position of the affected incisors, so it was decided to proceed with active orthodontic eruption. A Nance holding appliance and lower lingual arch were placed to maintain space in the arches. The maxillary first premolars and deciduous canines were extracted to relieve crowding and facilitate canine eruption in the maxillary arch. The permanent teeth were bonded in the maxillary arch after the eruption of the canines to align the teeth. An open coil spring was placed post alignment to create space for the impacted incisor. A closed eruption technique was planned as the tooth was apical to the mucogingival junction. Orthodontic vertical traction was applied 1-week post-surgery using an e-chain tied to a modified 0.018 SS wire containing a helix. Over the next 6 months, the progress of incisor eruption was evaluated monthly. Once sufficiently erupted, the crown was bonded with an incisor bracket to align the maxillary arch (Fig. 3). A lower right central incisor was extracted to relieve the crowding in the lower arch. The active treatment took 18 months. At the end of treatment, the upper and lower arches were aligned, there was a class 2 molar relation and class 1 canine relation (Fig. 4).

\section{Case 2}

An 11-year-old male in permanent dentition was reported with a chief complaint of missing upper front teeth. The patient had a convex profile and competent lips. On intraoral examination, the patient had a class 1 molar relationship bilaterally with missing maxillary right central and lateral incisors, retained deciduous canine, mandibular arch crowding, and retroclined maxillary left central and lateral incisors (Fig. 5). Routine panoramic radiograph revealed impacted incisors, unerupted canine, and a supernumerary tooth as well. Cone-beam computed tomography scan done to evaluate the shape and exact position of the supernumerary tooth revealed that it was lying between right central and lateral incisors. Cone-beam computed tomography scan also revealed the presence of dilaceration of impacted central incisor root which was curved distobuccally near the apical third (Fig. 6). The cause of impaction was established as an obstruction to the path of eruption due to supernumerary teeth.

The treatment plan was the removal of deciduous canine, supernumerary tooth, and surgical exposure of impacted incisors and unerupted canine followed by evaluation at a later date for all first premolar extraction depending on the soft tissue profile.

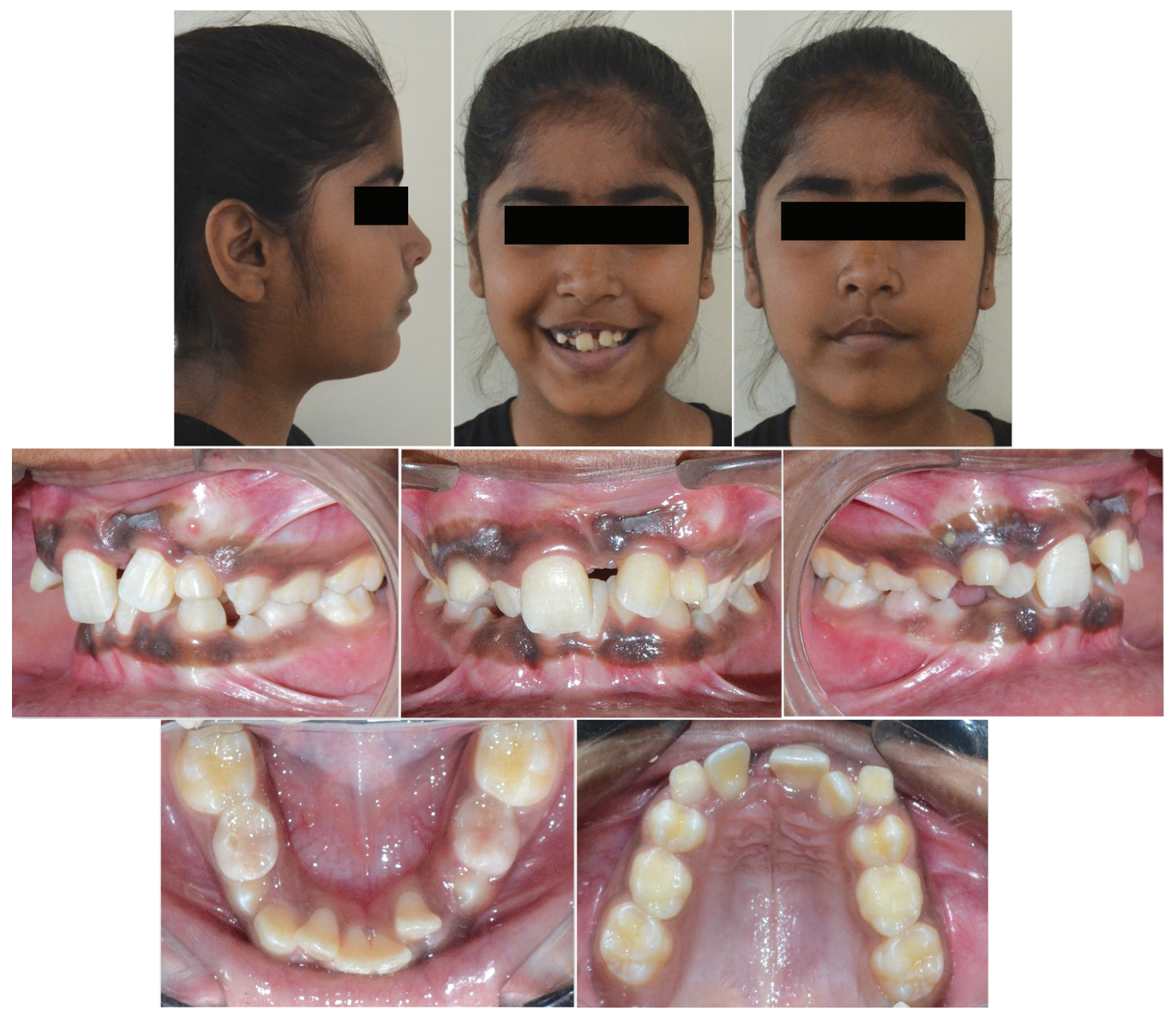

Fig. 1: Case 1: Pretreatment extraoral and intraoral photographs 


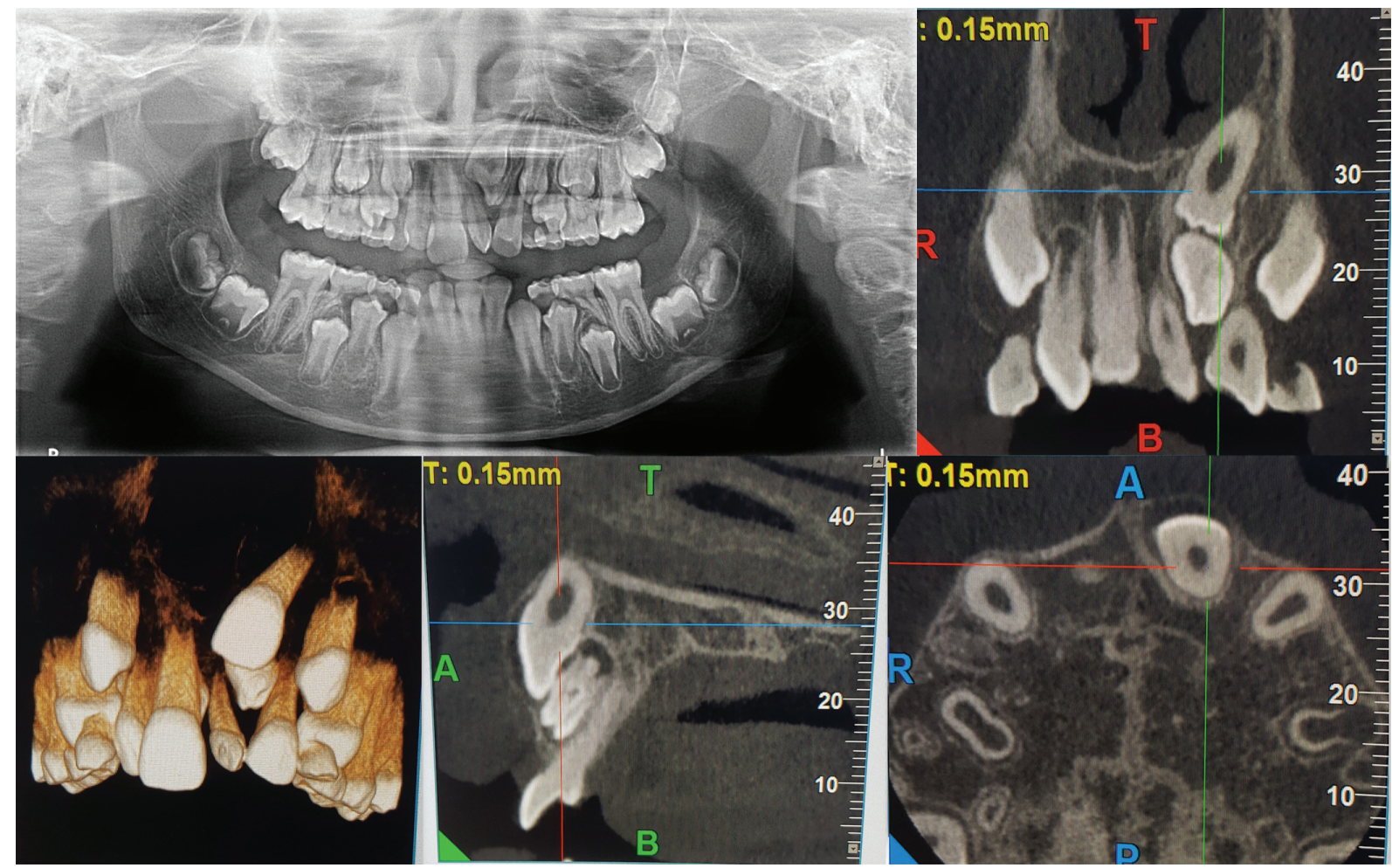

Fig. 2: Case 1: Pretreatment OPG and CBCT images

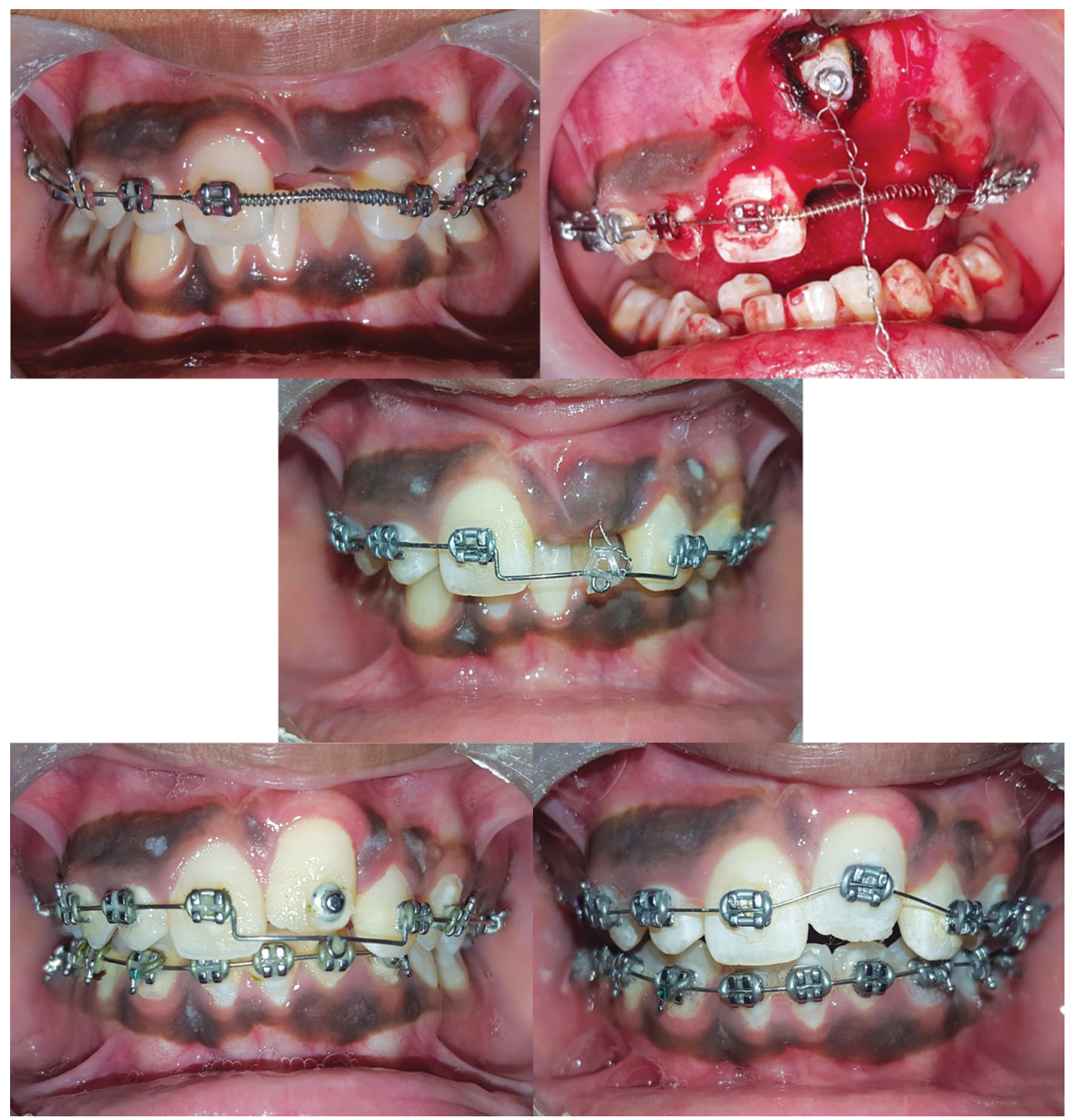

Fig. 3: Closed surgical technique for exposure of incisor followed by traction with e-chain on 0.018 ss wire 


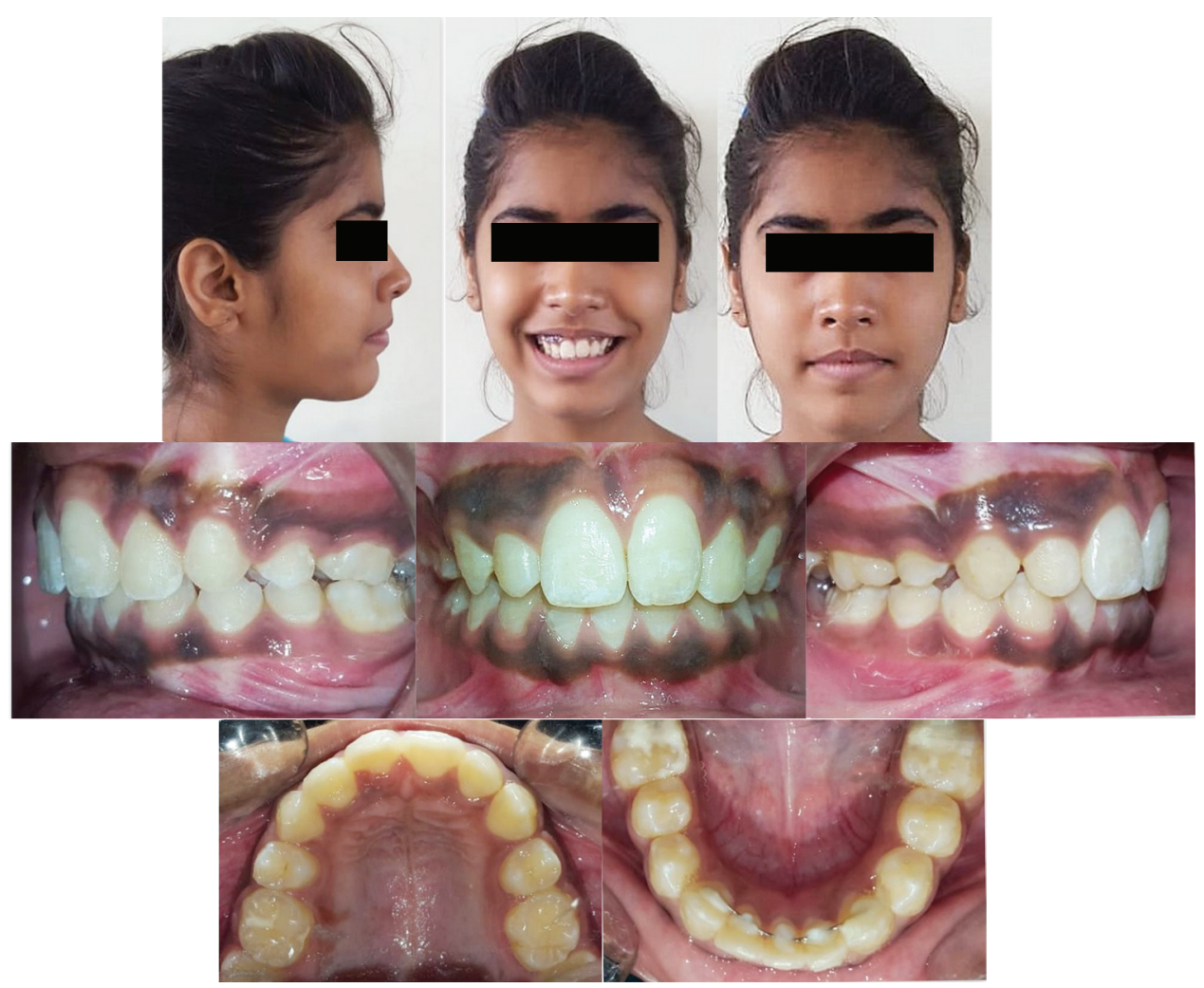

Fig. 4: Case 1: Posttreatment extraoral and intraoral photographs

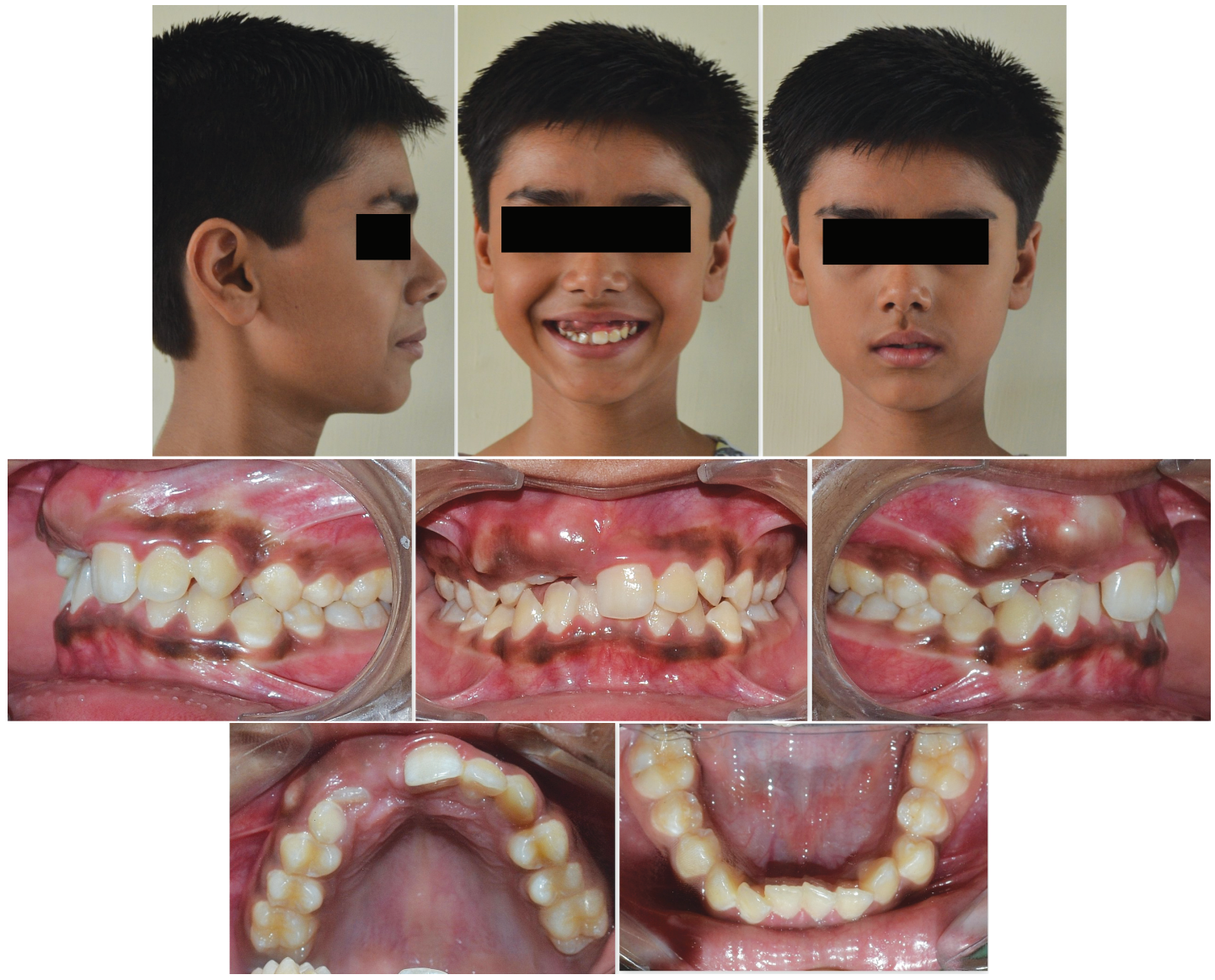

Fig. 5: Case 2: Pretreatment extraoral and intraoral photographs 
An open coil spring was placed post alignment to create space for the impacted incisor on an 018 round SS wire. An open eruption technique was planned as the tooth was below the mucogingival junction near the alveolar crest along with the extraction of deciduous canine and supernumerary teeth. The impacted incisor and unerupted canine were bonded at the same time as the exposure. Spontaneous eruption of the teeth occurred while space was maintained with an open coil NiTi spring. Over the next 3 months, the progress of incisor eruption was evaluated monthly. Once sufficiently erupted, the crown was bonded with an incisor bracket to align the maxillary arch (Fig. 7). Proximal stripping was done to relieve the crowding in the lower arch. The active treatment took 18 months. At the end of treatment, the upper and lower arches were aligned, there was a class 1 molar and class 1 canine relation (Fig. 8).

\section{Case 3}

A 7-year-old male in mixed dentition was reported with a chief complaint of missing upper front tooth. On intraoral examination, the patient had a class 1 molar relationship with a missing maxillary left central incisor. A bulge could be felt in the palate in the anterior region (Fig. 9). A routine panoramic radiograph revealed an impacted incisor. The lateral cephalogram revealed a dilaceration in the incisor with the crown displaced palatally. The CBCT showed a palatally impacted dilacerated central incisor with root placed labially (Fig. 10). As the patient was in mixed dentition, a $2 \times 4$ appliance was planned. There was adequate space for the incisor therefore initial alignment followed by surgical exposure was planned. For the surgical protocol, an open surgical procedure was selected because the crown was covered only with palatal mucosa. A vertical loop was fabricated that was pushed back and ligated to the button to aid in the eruption of the tooth (Fig. 11). Once the crown was brought into the oral cavity with traction, a piggyback $0.012 \mathrm{NiTi}$ wire on an $0.018 \mathrm{SS}$ wire was placed. After the alignment of the tooth in the arch, root torque was given in a $17 \times 25$ TMA wire to bring the root in the proper position. The active treatment took 18 months. At the end of treatment, the upper incisor was well aligned (Fig. 12).

\section{Discussion}

Treatment of impacted incisors requires careful treatment planning and a joint effort of an orthodontist and oral surgeon.

The choice of modality for treatment depends on several patient and dental factors. A comprehensive medical history should be taken to rule out any medical condition which may impact orthodontic and or surgical treatment. ${ }^{9}$ General management of impacted incisors includes removal of physical obstruction, creation of space, allowing spontaneous eruption, and surgical exposure with or without traction. ${ }^{2}$

In case 1 and case 2, supernumerary teeth were the cause of the impaction of incisors. While a similar protocol was followed in both cases, the impacted incisor failed to spontaneously erupt in case 1.

The presence of supernumerary/odontome is responsible for delayed eruption/impaction in $28-60 \%$ of cases. ${ }^{3}$ The optimal age of removal of the physical obstruction is unknown as it is influenced by the age at which the diagnosis is made. The spontaneous eruption is more likely if the associated supernumerary tooth is

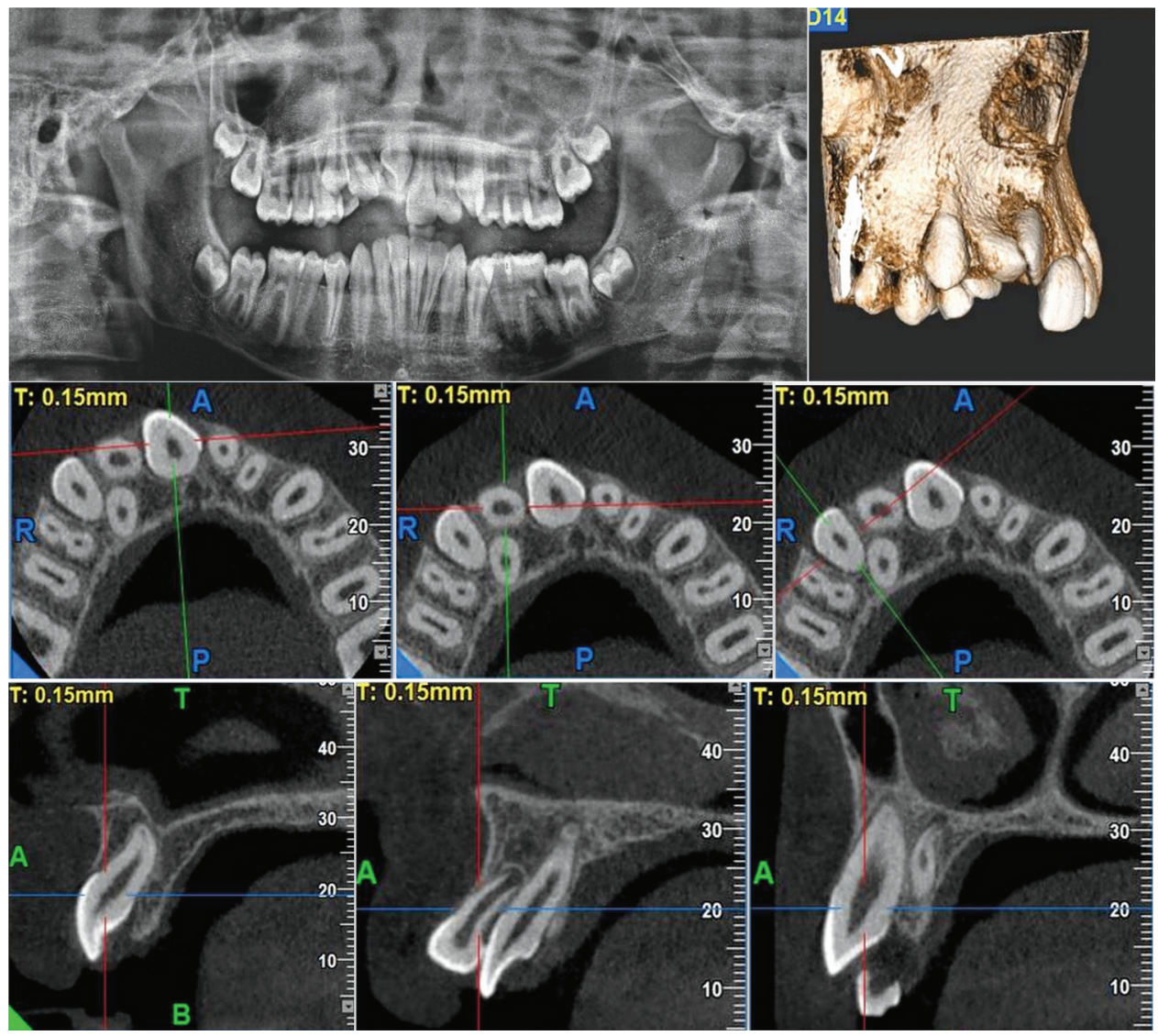

Fig. 6: Case 2: Pretreatment OPG and CBCT images 

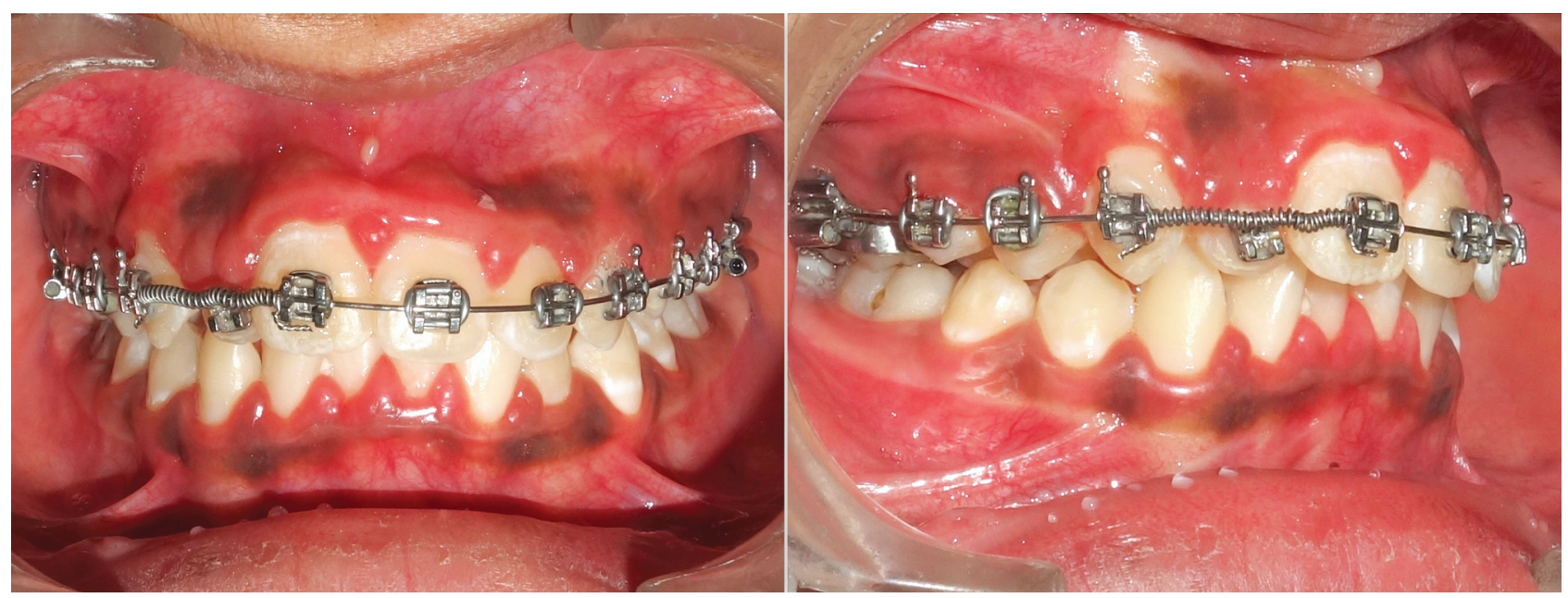

Fig. 7: Mid treatment photographs with open coil spring for creating space for 12

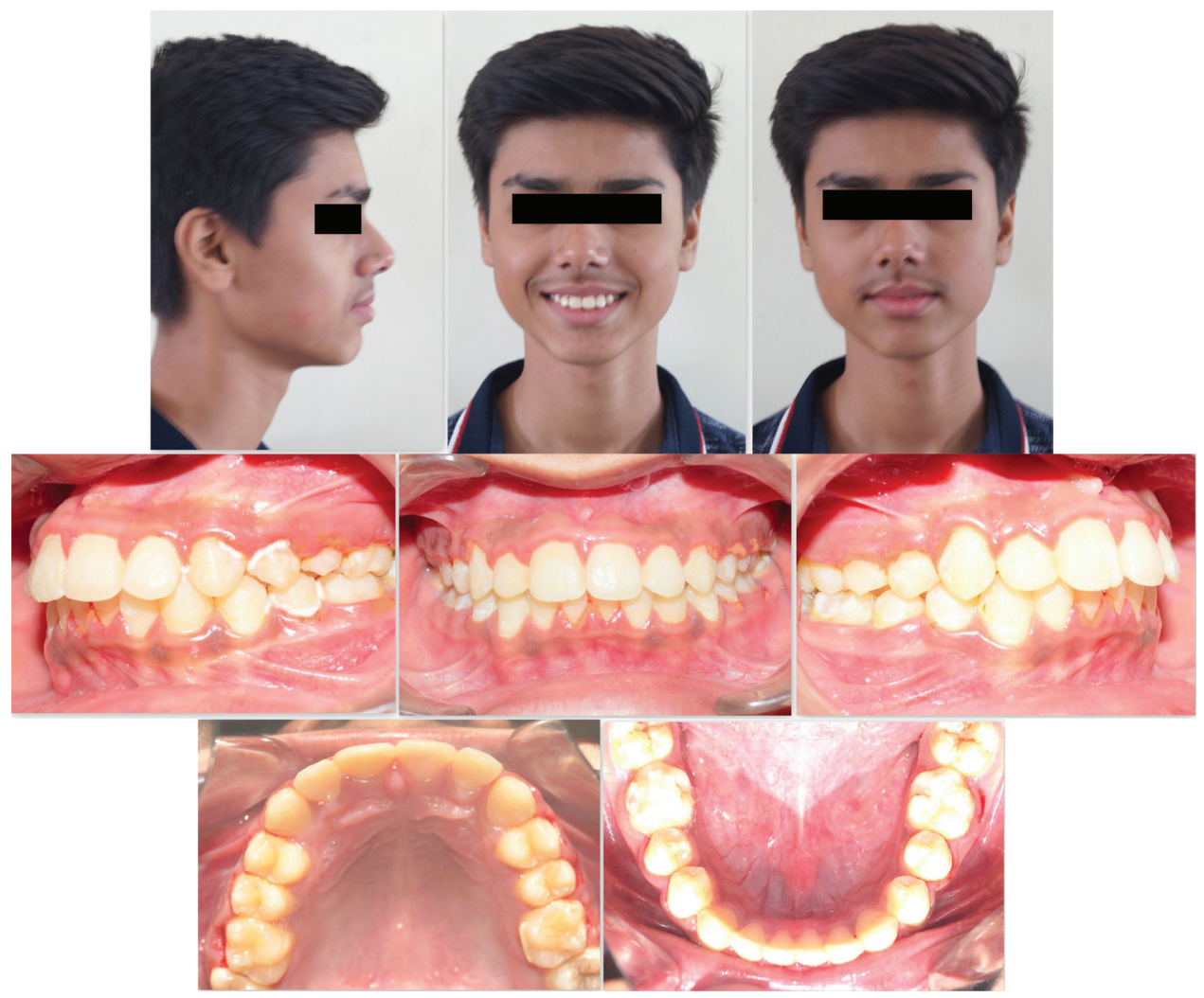

Fig. 8: Case 2: Posttreatment extraoral and intraoral photographs

removed between 8 years and 9 years of age. Studies have found that $49-91 \%$ of impacted incisors erupt spontaneously following removal of the supernumerary tooth but the time taken to erupt is variable, taking up to 18 months. ${ }^{10}$

The probability of eruption post removal of obstruction can be estimated by the vertical position, developmental stage, anatomy of the impacted incisor. In case 1, the impacted incisor was higher as compared to case 2 . This could be a reason for the spontaneous eruption post-exposure in the second case. It was observed by Smailiene et al. that $28.6 \%$ of unilaterally impacted permanent maxillary incisors erupted spontaneously when their initial position was at the level of the apical third of the root of the contralateral erupted maxillary incisor. ${ }^{11}$ Studies done to find an association between the eruption of unerupted maxillary incisors and their stage of root development have contrasting views. According to Mason et al., ${ }^{12}$ an impacted incisor is more likely to erupt if it has immature roots while Di Base et al. found no association between the same. ${ }^{13}$ In a patient $<9$ years of age eruption is monitored for a period of 9-12 months after the removal of obstruction and creation of space.

As treatment of an impacted incisor requires orthodontic and surgical intervention, a judgment should be made about the ability of the patient to undergo treatment.

In patients $>9$ years of age, surgical exposure and bonding of attachment are considered at the time of removal of physical obstruction. In case 1, patient had undergone the extraction of 


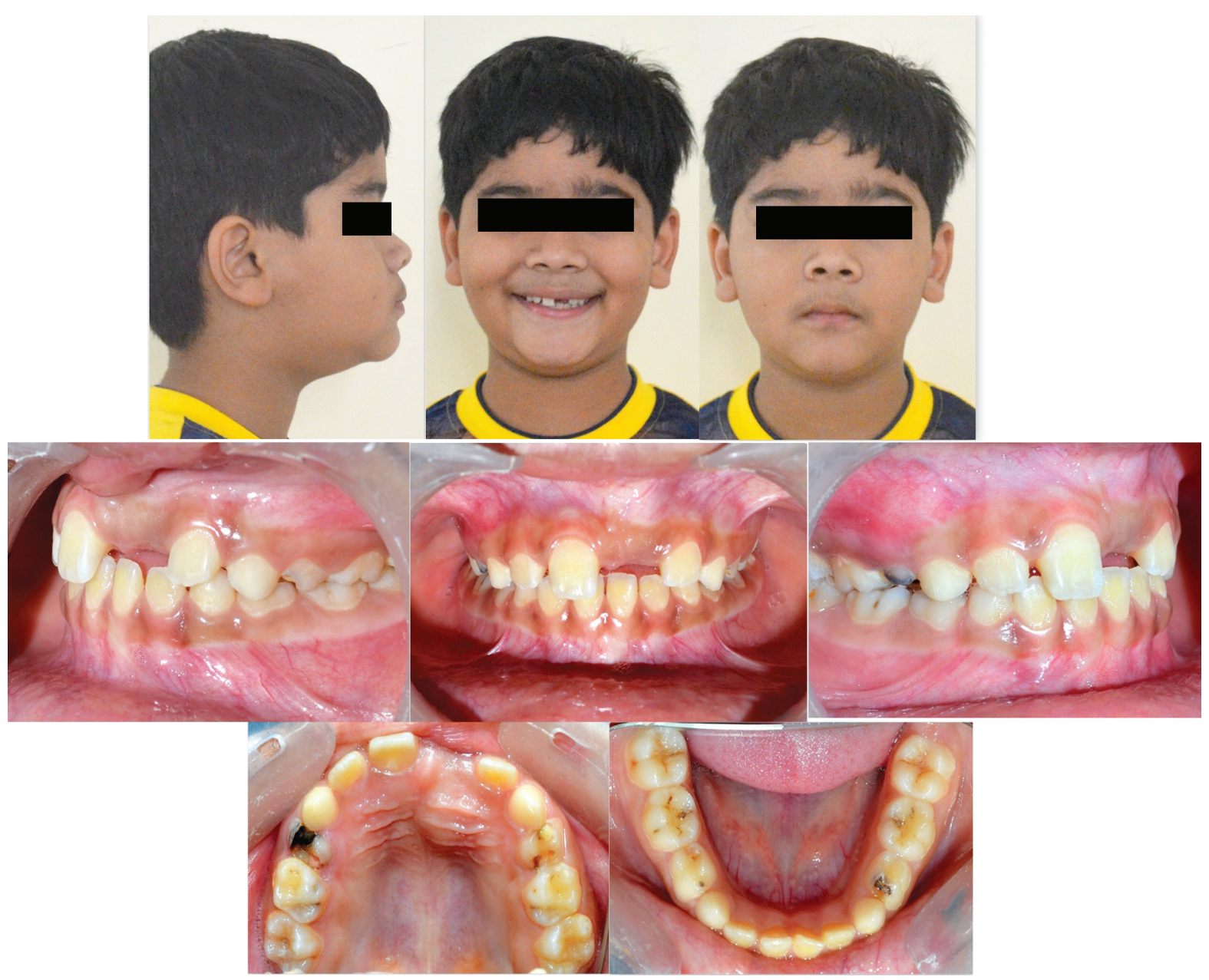

Fig. 9: Case 3: Pretreatment extraoral and intraoral photographs

the supernumerary before orthodontic consultation. Therefore, an additional surgical procedure was required once the impacted tooth failed to erupt spontaneously. In case 2, the attachments were bonded at the same time as the exposure of the tooth.

The orthodontic attachment should be bonded at the time of removal of physical obstruction to avoid repeated anesthesia. The chances of an eruption of impacted tooth increase to $90 \%$ when surgical exposure is combined with orthodontic traction. However, the duration of treatment is influenced by the height of impaction. ${ }^{14}$

Open or closed eruption technique can be selected to expose the impacted tooth and apply orthodontic traction.

The open technique involves an elliptical incision in soft tissue overlying the impacted tooth. This technique is useful only if the impacted tooth occupies a very superficial position beneath the mucosa or if you can retain adequate width of attached gingiva after exposure. Open exposure allows a dry field for bonding, planning the direction of traction. However, the technique is known to result in increased crown length and poor esthetics. ${ }^{15}$ This technique was selected in cases 2 and 3 as the teeth were very superficial, i.e., just below the gingiva.

In the closed eruption technique, a full-thickness flap is raised, an attachment is bonded and the flap is replaced. The technique is beneficial for cases with deep impaction of a very highly positioned tooth. Therefore, this technique was selected in case 1. A clinical photograph at the time of bonding the attachment is useful to plan the direction of traction. This technique has the advantage of better esthetics as it preserves the attached gingiva. ${ }^{14}$

Dilacerated teeth pose a challenge in treatment. The extent of dilacerations, inclination of the crown and root, and the quality and quantity of the alveolar bone at the site where the tooth will be moved determine whether attempting orthodontic alignment is feasible. ${ }^{3}$ The direction of displacement of the crown, height of the crown influences the type of surgical intervention. In case of an unfavorable labial root dilaceration, elective root filling and apicoectomy may be required. Case 3 presented with dilacerations with the crown displaced palatally. The incisor was superficial, just below the gingiva. Therefore, an open exposure was planned.

Different options may be considered in the case of ankylosed maxillary incisors. In case of an impacted tooth with minor infraocclusion composite build-up may be considered. The tooth may be repositioned by surgical dislodgement, osteotomy, or distraction osteogenesis. ${ }^{16}$

Removal of impacted incisors may be considered in cases of significant dilacerations or ankylosis with infraocclusion. Patients with impacted incisors are generally diagnosed at a young age. An unfavorably impacted tooth requires prolonged space maintenance following removal. This may result in a reduction in bone height and width compromising future implant placement. In such cases, the tooth may be left in situ till definitive treatment is started. ${ }^{17}$ None of the three cases presented with unfavorable dilacerations 


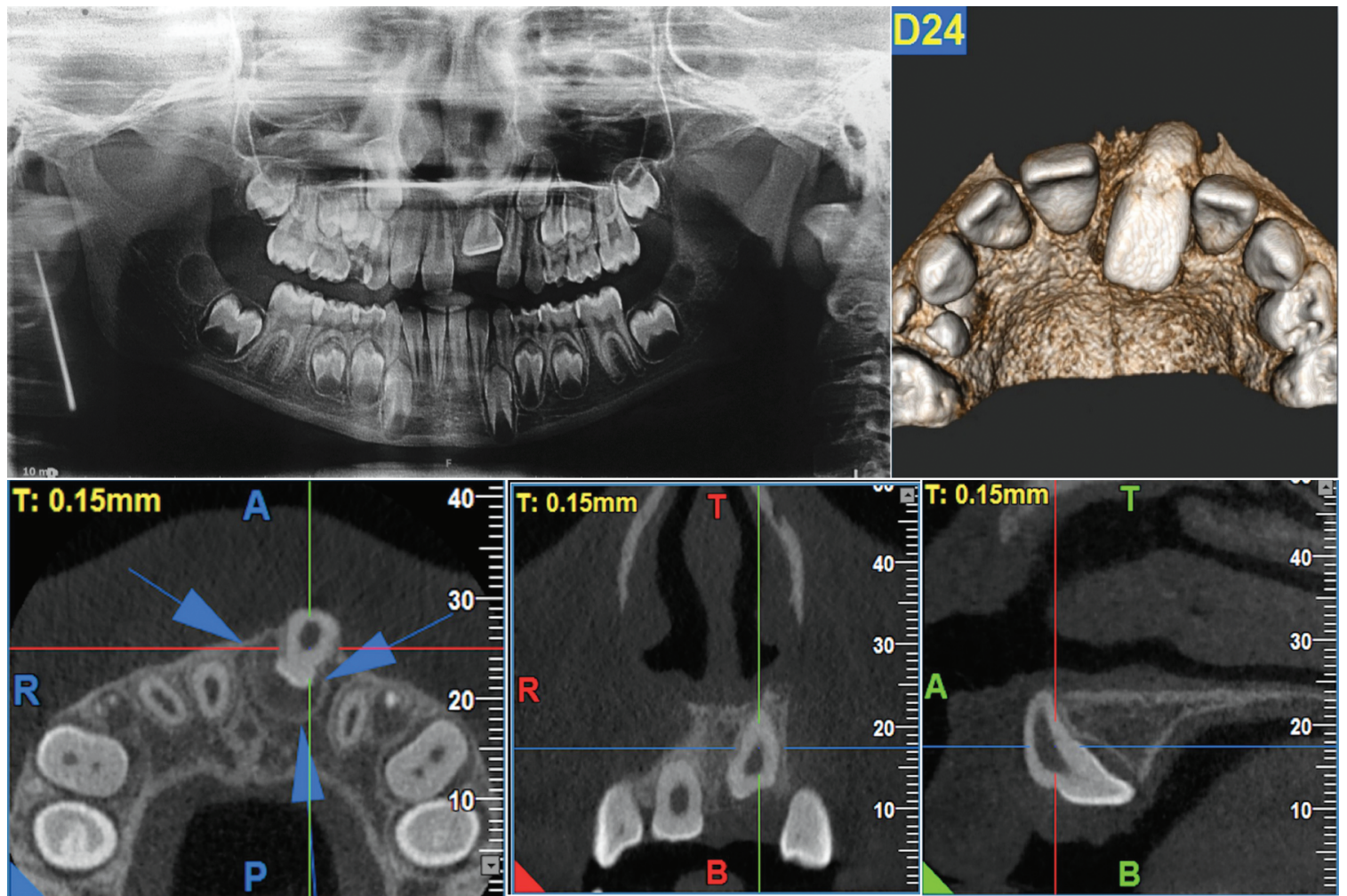

Fig. 10: Case 3: Pretreatment OPG and CBCT images

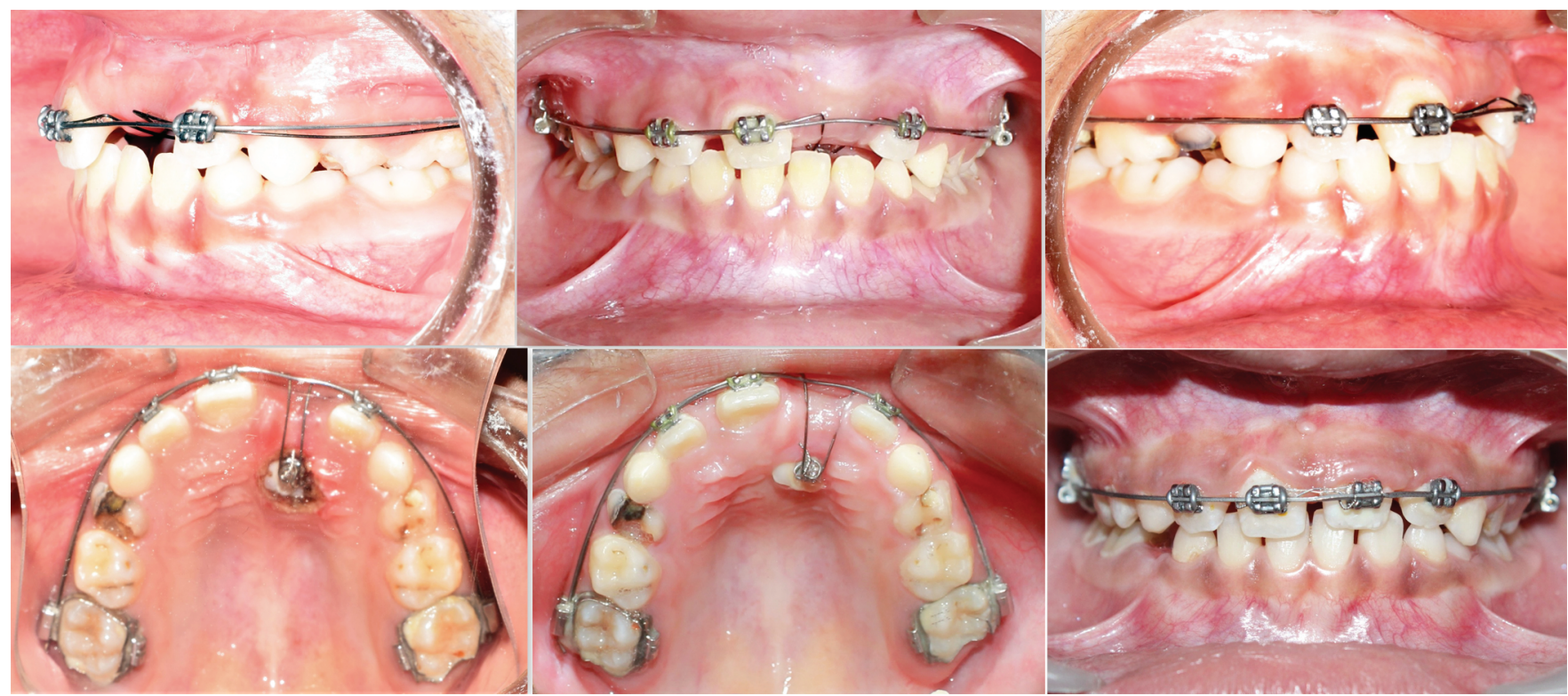

Fig. 11: Mid treatment photographs with $2 \times 4$ appliance followed by open exposure and vertical loop for traction

or ankyloses. The treatment duration was affected by the height of impaction and the type of dilacerations.

Autogenous tooth transplantation was first documented in 1954 by ML Hale. It involves the placement of the patient's own vital tooth with preserved periodontium. In case of an unfavorably impacted incisor, a lower second premolar may be transplanted in its place. The major advantage of this technique is that a prosthesis is not needed. ${ }^{18}$ Czochrowska stated that with careful case selection the transplanted tooth has a good long-term survival with the maintenance of the alveolar process. But there are still some disadvantages as the transplanted tooth requires extensive restorative work, which may result in interference of palatal cusp and external root resorption with loss of the tooth. ${ }^{3}$

\section{Conclusion}

Impaction of maxillary anterior teeth can be a challenging orthodontic problem. 


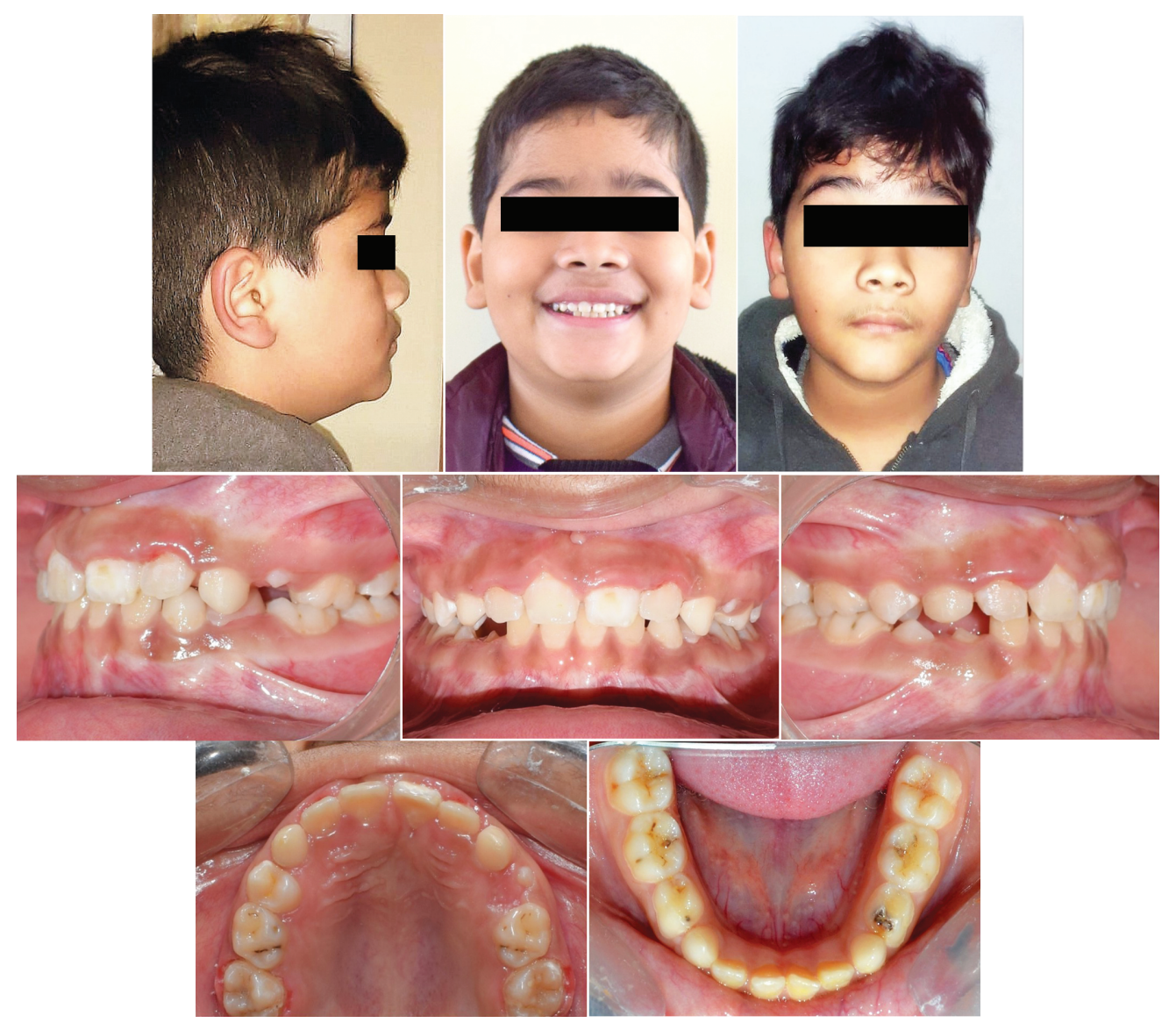

Fig. 12: Case 3: Posttreatment extraoral and intraoral photographs

Early diagnosis of delayed eruption is important and it is made based on clinical and radiographic findings.

The treatment of an unerupted tooth will depend upon its state, position, and presence of enough space in the dental arch to accommodate. In the cases described in the present case series, an interceptive approach resulted in a satisfactory approach.

\section{References}

1. Tanki JZ, Naqash TA, Gupta A, et al. Impacted maxillary incisors: causes, diagnosis and management. J Dent Med Sci 2013;5(2):41--45. DOI: 10.9790/0853-0524145.

2. Yaqoob O, O'Neill J, Gregg T, et al. Management of unerupted maxillary incisors. (April 2010).

3. Seehra J, Yaqoob O, Patel S, et al. National clinical guidelines for the management of unerupted maxillary incisors in children. Br Dent J 2018;224(10):779-785. DOI: 10.1038/sj.bdj.2018.361.

4. Khera AK, Rohilla A, Tandon P, et al. Orthodontic management of impacted central incisor: a clinical challenge. J Indian Orthod Soc 2017;51(1):46-50. DOI: 10.4103/0301-5742.199249.

5. Huber KL, Suri L, Taneja P. Eruption disturbances of the maxillary incisors: a literature review 1. J Clin Pediatr Dent 2008;32(3):221-230. DOI: 10.17796/jcpd.32.3.m175g328l100x745.

6. de Amorim CS, Americano GCA, Moliterno LFM, et al. Frequency of crown and root dilaceration of permanent incisors after dental trauma to their predecessor teeth. Dent Traumatol 2018;34(6):401405. DOI: 10.1111/edt.12433.

7. Pavoni C, Mucedero M, Laganà G, et al. Impacted maxillary incisors: diagnosis and predictive measurements. Ann Stomatol (Roma) 2012;III(3/4):100-105.
8. Jacobs SG. Radiographic localization of unerupted maxillary anterior teeth using yhe vertical tube shift technique: the history and application of the method with some case reports. Am J Orthod Dentofac Orthop 1999;116(4):415-423. DOI: 10.1016/S08895406(99)70226-X.

9. Patel A, Burden DJ, Sandler J. Medical disorders and orthodontics. J Orthod 2009;36(sup1):1-21. DOI: 10.1179/14653120723346.

10. Leyland $L$, Batra $P$, Wong $F$, et al. A retrospective evaluation of the eruption pf impacted permanent incisors after extraction of supernumerary teeth. J Clin Paedriatr Dent 2006;30:225-231.

11. Smailiene D, Sidlauskas A, Bucinskiene J. Impaction of the central maxillary incisor associated with supernumerary teeth: initial position and spontaneous eruption timing. Stomatologija 2006;8(4):103-107.

12. Mason C, Azam N, Holt RD, et al. A retrospective study of unerupted maxillary incisors associated with supernumerary teeth. $\mathrm{Br} J$ Oral Maxillofac Surg 2000;38(1):62-65. DOI: 10.1054/bjom.1999. 0210.

13. Di Biase DD. The effect of variations in tooth morphology and position on eruption. Dent Pract Dent Rec 1971;22(3):95-108.

14. Management of unerupted incisors 2016.

15. Vanarsdall RL, Corn H. Soft-tissue management of labially positioned unerupted teeth. july 1977. Am J Orthod Dentofacial Orthop 2004;125(3):284-293. DOI: 10.1016/j.ajodo.2003.10.003.

16. Malmgren B. Decoronation: how, why and when? J Calif Dent Assoc 2000;28(11):846-854.

17. Kokich VG, Crabill KE. Managing the patient with missing or malformed maxillary central incisors. Am J Orthod Dentofacial Orthop 2006;129(4 Suppl):S55-S63. DOI: 10.1016/j.ajodo.2005.11.007.

18. Clokie CM, Yau DM, Chano L. Autogenous tooth transplantation: an alternative to dental implant placement? J Can Dent Assoc 2001;67(2):92-96. 